\title{
Controllability of Noncompact-semigroup Fractional Impulsive Neutral Functional Inclusions in Banach Spaces
}

\author{
Zhixin Tai \\ College of Engineering, Bohai University, 121013 Jinzhou, P.R.China \\ taizhixin01@163.com
}

Keywords: Controllability; Measure of no compactness; Fixed point; Topological degree

\begin{abstract}
In this work, by employing the Hausdorff measure of no compactness, controllability of fractional impulsive neutral functional inclusions with a noncom pact semi group in a Banach space has been addressed where a multifunction is of Caratheodory type. Sufficient conditions for the controllability are established using topological degree theory for condensing operators.
\end{abstract}

\section{Introduction and Preliminaries.}

Theory of measure of no compactness has found various applications in the existence of solutions of systems [1,2] and inclusions [3] in Banach spaces. Up to now, controllability analysis is not available for fractional impulsive neutral functional inclusions with a noncom pact semi group in a Banach space using measure of no compactness whereby in this paper, controllability of the system has been presented using topological degree theory for condensing operators in terms of Hausdorff measure of no compactness.

Consider the following fractional impulsive neutral functional inclusions with a noncompact semigroup:

$$
\begin{aligned}
& \frac{d^{q}}{d t^{q}}\left[x(t)-g\left(t, x_{t}\right)\right] \in(A x)(t)+(B u)(t)+F\left(t, x_{t}\right), t \in J=[0, b], t \neq \tau_{k}, k=1,2, \cdots, m ; \\
& \left.\Delta x\right|_{t=\tau_{k}}=I_{k}\left(x\left(\tau_{k}^{-}\right)\right), k=1,2, \cdots, m ; \\
& x_{0}:=\phi \in B_{v}
\end{aligned}
$$

where the state $\mathrm{x}(\cdot)$ belongs to Banach space E endowed with the norm $\|\cdot\|, 0<q<1$. The control function $\mathrm{u}(\cdot)$ takes values in a Banach space $L^{2}(J, U)$ of admissible control functions. Operator $\mathrm{A}$ generates a strongly continuous noncompact semigroup of bounded linear operators $\mathrm{T}(\mathrm{t})$ in $\mathrm{E}$. B is a bounded linear operator from a Banach space U into E. $\left.\Delta x\right|_{t=\tau_{k}}=x\left(\tau_{k}^{+}\right)-x\left(\tau_{k}^{-}\right)$, for all $\mathrm{k}=1,2, \cdots, \mathrm{m}$, $0=\tau_{0}<\tau_{1}<\tau_{2}<\cdots<\tau_{m}<\tau_{m+1}=b$. Let $x_{t}(\cdot)$ denote $x_{t}(\theta):=x(t+\theta), \theta \in(-\infty, 0]$.

Assume $v:(-\infty, 0] \rightarrow(0,+\infty)$ is a continuous function satisfying $l=\int_{-\infty}^{0} v(t) d t<\infty$. Banach space $\left(B_{v},\|\cdot\|_{B_{v}}\right)$ induced by function $v$ is defined as follows.

$B_{v}:=\{\varphi:(-\infty, 0] \rightarrow E:$ for any $\mathrm{c}>0, \varphi(\theta)$ is a bounded and measurable function on [-c, 0$]$, and

$$
\left.\int_{-\infty}^{0} v(s) \cdot \sup _{s \leq \theta \leq 0}|\varphi(\theta)| d s<+\infty\right\}
$$

endowed with norm $\|\varphi\|_{B_{v}}:=\int_{-\infty}^{0} v(s) \cdot \sup _{s \leq \theta \leq 0}|\varphi(\theta)| d s$. 
Let us define the space $B_{v}^{\prime}:=\left\{\varphi:(-\infty, b] \rightarrow E: \varphi_{k} \in C\left(J_{k}, X\right)\right.$ and exists $\varphi\left(t_{k}^{-}\right)$and $\varphi\left(t_{k}^{+}\right)$with

$$
\left.\varphi\left(t_{k}\right)=\varphi\left(t_{k}^{-}\right), \varphi_{0}=\phi \in B_{v}, k=0,1, \cdots, m\right\}
$$

where $\varphi_{k}$ is the restriction of $\varphi$ to $J_{k}, J_{0}:=\left[0, t_{1}\right], J_{k}:=\left(t_{k}, t_{k+1}\right], k=1,2, \cdots, m$.

Denote by $\|\cdot\|_{B_{v}^{\prime}}$ a seminorm in the space $B_{v}^{\prime}$ defined by

$$
\|\varphi\|_{B_{v}^{\prime}}=\|\varphi\|_{B_{v}}+\sup \{|\varphi(s)|: s \in[0, b]\}, \text { for } \varphi \in B_{v}^{\prime}
$$

Define Banach space $\left(B_{v}^{\prime \prime},\|\cdot\|_{B_{v}}\right)$ induced by the space $B_{v}^{\prime}$

$$
B_{v}^{\prime \prime}:=\left\{\varphi \in B_{v}^{\prime}: 0=\varphi_{0} \in B_{v}\right\} \text { with norm }\|\varphi\|_{B_{v}^{\prime}}=\sup \{|\varphi(s)|: s \in[0, b]\}
$$

Let space $B_{r}:=\left\{\varphi \in B_{v}^{\prime \prime}:\|\varphi\|_{B_{v}^{\prime}} \leq r\right\}$ for some $r>0$, then $B_{r}$, for each $r$, is a bounded, closed convex subset in $\mathrm{X}$.

Before proceeding, we recall some supporting definitions and properties which will help in developing our main results in the next section.

Definition 1.1 A function $x:(-\infty, b] \rightarrow E$ is called a mild solution of the system Eq.(1)-Eq.(3), if the impulsive condition $\left.\Delta x\right|_{t=\tau_{k}}=I_{k}\left(x\left(\tau_{k}^{-}\right)\right), k=1,2, \cdots, m$, is verified, the restriction of $x(\cdot)$ to the interval $J_{k}(k=0,1, \cdots, m)$ is continuous and the following integral equation holds: for $t \in J$,

$$
\begin{aligned}
x(t)= & T(t)[\phi(0)-g(0, \phi)]+g\left(t, x_{t}\right)+\frac{1}{\Gamma(q)} \int_{0}^{t}(t-s)^{q-1} T(t-s)\left[A g\left(s, x_{s}\right)+(B u)(s)+f(s)\right] d s \\
& +\sum_{0<\tau_{k}<t} T\left(t-\tau_{k}\right) I_{k}\left(x\left(\tau_{k}^{-}\right)\right)
\end{aligned}
$$

where $f \in P_{F}(x):=\left\{f \in C(J ; E): f(t) \in F\left(t, x_{t}\right)\right.$, a.e. $\left.t \in J\right\}$ and $u \in L^{2}(J ; U)$

Definition 1.2 The system Eq.(1)-Eq.(3) is said to be controllable on the interval $J$ if for every initial function $\phi \in C((-\infty, 0], E)$ and $x_{1} \in E$, there exists a control $u \in L^{2}(J, U)$ such that mild solution $x(t)$ of Eq.(1)-Eq.(3) satisfies $x(b)=x_{1}$

Lemma 1.1 Assume $x \in B_{v}^{\prime}$, then for $t \in J, x_{t} \in B_{v}$.

Moreover, $l|x(t)| \leq\left\|x_{t}\right\|_{B_{v}} \leq\|\phi\|_{B_{v}}+l \sup _{s \in[0, t]}|x(s)|$

Lemma 1.2 Let $\mathrm{V}$ be a bounded open neighbourhood of zero in Banach space $\varepsilon$ and $F: \bar{V} \rightarrow K_{v}(\varepsilon)$ an u.s.c. $\beta$-condensing multimap satisfying the boundary condition $x \notin \lambda F(x)$ for all $x \in \partial V$ and $0<\lambda<1$, where $K_{v}(X)$ denotes the collection of all nonempty compact-convex subsets of $\mathrm{X}$. Then $\operatorname{Fix}(F) \neq \phi$.

Lemma 1.3 Let $\Theta: C(J ; E) \rightarrow C(J ; E)$ be an abstract operator satisfying the following conditions: (S1) there exists $D \geq 0$ such that $\sim \Theta f-\Theta g\left\|_{C} \leq D\right\| f-g \|_{C}, \forall f, g \in C(J ; E)$

(S2) for each compact set $K \subset E$ and sequence $\left\{f_{n}\right\} \subset C(J ; E)$ such that $\left\{f_{n}(t)\right\} \subset K$ for a.e. $t \in J$, the weak convergence $f_{n} \rightarrow f_{0}$ implies $\sim f_{n} \rightarrow \Theta f_{0}$.

Then the composition operator $\Theta \circ P_{F}: C(J ; E) \rightarrow C(J ; E)$ is a u.s.c. multimap with compact values. 
Lemma 1.4 Cauchy operator $G: C(J ; X) \rightarrow C(J ; X)$ defined by $(G f)(t):=\frac{1}{\Gamma(q)} \int_{0}^{t}(t-s)^{q-1} T(t-s) f(s) d s$ satisfies properties (S1)-(S2).

Lemma 1.5 Let $\mathrm{E}$ be a Banach space and $\Phi:[0, b] \rightarrow P(E)$ an integrable, integrably bounded multifunction such that $X_{E}(\phi(t)) \leq p(t)$ for a.e. $t \in[0, b]$ where $p(\cdot) \in C\left(J, R_{+}\right)$.

Then $X_{E}\left(\frac{1}{\Gamma(q)} \int_{0}^{t}(t-s)^{q-1} \phi(s) d s\right) \leq \frac{1}{\Gamma(q)} \int_{0}^{t}(t-s)^{q-1} p(s) d s$ for all $t \in[0, b], 0<q<1$

\section{Main Results}

To investigate the controllability of system Eq.(1)-Eq.(3), we assume the following conditions:

(H1) Operator A is the infinitesimal generator of a noncompact semigroup of bounded linear operators $T(\mathrm{t})$ in X satisfying $\|T(t)\| \leq \tilde{M}$ for some $\tilde{M} \geq 1$ when $t \geq 0$.

(H2) The linear operator $W: L^{2}(J ; U) \rightarrow E$, defined by $W u=\frac{1}{\Gamma(q)} \int_{0}^{b}(b-s)^{q-1} T(b-s)(B u)(s) d s$,

has an invertible operator $W^{-1}$ taking values in $L^{2}(J, U) \backslash \operatorname{Ker} W$ and there exist positive constants $\tilde{M}_{1}, \tilde{M}_{2}$ such that $\|B\| \leq \tilde{M}_{1},\left\|W^{-1}\right\| \leq \tilde{M}_{2}$.

(H3) There exist positive constants $\gamma_{g}, \gamma_{g}^{\prime}, \gamma_{k}$ such that the following Lipschitz conditions hold:

$$
\begin{aligned}
& \left\|g\left(t, x_{t}\right)-g\left(t, \hat{x}_{t}\right)\right\| \leq \gamma_{g}\left\|x_{t}-\hat{x}_{t}\right\|_{B_{v}} \quad, \quad \quad\left\|A g\left(t, x_{t}\right)-A g\left(t, \hat{x}_{t}\right)\right\| \leq \gamma_{g}^{\prime}\left\|x_{t}-\hat{x}_{t}\right\|_{B_{v}} \\
& \left\|I_{k}(x(t))-I_{k}(\hat{x}(t))\right\| \leq \gamma_{k}\|x(t)-\hat{x}(t)\|
\end{aligned}
$$

(H4)The multivalued nonlinearity $F: J \times B_{v} \rightarrow K_{v}(E)$ satisfies the following conditions:

$\left(\mathrm{H}^{\prime}\right) \quad F\left(\cdot, x_{t}\right): J \rightarrow K_{v}(E)$ admits a measurable selection for every $x_{t} \in B_{v}$.

$(\mathrm{H} 4 ") \quad F(t, \cdot): B_{v} \rightarrow K_{v}(E)$ is u.s.c.for a.e. $t \in J$

(H4"') there exist a function $h(\cdot) \in C\left(J ; R_{+}\right)$and a nondecreasing function $\psi: R_{+} \rightarrow R_{+}$such that $\left\|F\left(t, x_{t}\right)\right\| \leq h(t) \psi\left(\left\|x_{t}\right\|_{B_{v}}\right)$ for a.e. $t \in J$.

(H5) there exists a function $k(\cdot) \in L_{+}^{1}(J)$ such that $X_{E}(F(t, M)) \leq k(t) X_{E}(M)$ for every bounded set $M \subset B_{v}^{\prime \prime}$.

(H6) there exists a function $X(\cdot) \in L_{+}^{1}(J)$ such that $X_{U}\left(W^{-1}\left(M_{1}\right)(t)\right) \leq X(t) X_{E}\left(M_{1}\right)$ for every bounded set $M_{1} \subset E$

In view of hypothesis $(\mathrm{H} 2)$, for an arbitrary function $x(\cdot)$, the control is defined as follows:

$$
\begin{aligned}
& u(t)=W^{-1}\left[x_{1}-T(b)(\phi(0)-g(0, \phi))-g\left(b, x_{b}\right)-\frac{1}{\Gamma(q)} \int_{0}^{b}(b-s)^{q-1} T(b-s)\left(A g\left(s, x_{s}\right)+f(s)\right) d s\right. \\
& \left.-\sum_{k=1}^{m} T\left(b-\tau_{k}\right) I_{k}\left(x\left(\tau_{k}^{-}\right)\right)\right](t), \quad f \in P_{F}(x)
\end{aligned}
$$

In what follows, it suffices to show that when using this control the multi-valued operator $\Gamma$ defined by 


$$
(\Gamma x)(t)=\left\{\begin{array}{l}
\phi(t), t \in(-\infty, 0] \\
\left\{z(t) \in X: z(t)=T(t)(\phi(0)-g(0, \phi))+g\left(t, x_{t}\right)+\frac{1}{\Gamma(q)} \int_{0}^{t}(t-s)^{q-1} T(t-s)\left(A g\left(s, x_{s}\right)\right.\right. \\
\left.+(B u)(s)+f(s)) d s+\sum_{0<\tau_{k}<t} T\left(t-\tau_{k}\right) I_{k}\left(x\left(\tau_{k}^{-}\right)\right), \quad f \in P_{F}(x)\right\}, \quad t \in J
\end{array}\right.
$$

has a fixed point $x(\cdot)$ from which it follows this fixed point is a mild solution of the system Eq.(1)Eq.(3). Clearly, $x(b)=(\Gamma x)(b)=x_{1}$, which concludes that the system is controllable.

Let $x(t)=y(t)+\hat{\phi}(t), t \in(-\infty, b]$, where $\hat{\phi}$ is taken as $\phi(t)$ for $t \in(-\infty, 0]$ while for $t \in J$, defined as $T(t) \phi(0)$.

Define the operator $\Omega$ by

$$
(\Omega y)(t)=\left\{\begin{array}{l}
0, t \in(-\infty, 0] \\
\left\{z^{\prime}(t) \in X: z^{\prime}(t)=-T(t) g(0, \phi)+g\left(t, y_{t}+\hat{\phi}_{t}\right)+\frac{1}{\Gamma(q)} \int_{0}^{t}(t-s)^{q-1} T(t-s)\left(A g\left(s, y_{s}+\hat{\phi}_{s}\right)\right.\right. \\
\left.+(B u)(s)+f(s)) d s+\sum_{0<\tau_{k}<t} T\left(t-\tau_{k}\right) I_{k}\left(y\left(\tau_{k}^{-}\right)+\hat{\phi}\left(\tau_{k}^{-}\right)\right), \quad f \in P_{F}(y)\right\}, \quad t \in J
\end{array}\right.
$$

Obviously, the operator $\Omega$ has a fixed point if and only if operator $\Gamma$ has a fixed point. In the sequel, we shall consider the operator $\Theta: C(J ; E) \rightarrow C(J ; E)$ defined by

$$
\begin{aligned}
(\Theta f)(t):= & -T(t) g(0, \phi)+g\left(t, y_{t}+\hat{\phi}_{t}\right)+\frac{1}{\Gamma(q)} \int_{0}^{t}(t-s)^{q-1} T(t-s)\left(A g\left(s, y_{s}+\hat{\phi}_{s}\right)\right. \\
& +(B u)(s)+f(s)) d s+\sum_{0<\tau_{k}<t} T\left(t-\tau_{k}\right) I_{k}\left(y\left(\tau_{k}^{-}\right)+\hat{\phi}\left(\tau_{k}^{-}\right)\right)
\end{aligned}
$$

Where, $u$ takes values in the equation (4).

Lemma 2.1 The operator $\Theta$ satisfies properties (S1)-(S2).

Proof. In view of alternative representation of operator $\Theta$ of the form

$$
\begin{aligned}
(\Theta f)(t):= & -T(t) g(0, \phi)+g\left(t, y_{t}+\hat{\phi}_{t}\right)+\frac{1}{\Gamma(q)} \int_{0}^{t}(t-s)^{q-1} T(t-s)\left(A g\left(s, y_{s}+\hat{\phi}_{s}\right) d s\right. \\
& +\sum_{0<\tau_{k}<t} T\left(t-\tau_{k}\right) I_{k}\left(y\left(\tau_{k}^{-}\right)+\hat{\phi}\left(\tau_{k}^{-}\right)\right)+(G f)(t)+\left(\Theta_{1} f\right)(t)
\end{aligned}
$$

Where

$$
\begin{aligned}
\left(\Theta_{1} f\right)(t):= & \frac{1}{\Gamma(q)} \int_{0}^{t}(t-s)^{q-1} T(t-s) B W^{-1}\left[x_{1}-T(b)(\phi(0)-g(0, \phi))-g\left(b, y_{b}+\hat{\phi}_{b}\right)\right. \\
& \left.-\frac{1}{\Gamma(q)} \int_{0}^{b}(b-s)^{q-1} T(b-s)\left(A g\left(s, y_{s}+\hat{\phi}_{s}\right)+f(s)\right) d s-\sum_{k=1}^{m} T\left(b-\tau_{k}\right) I_{k}\left(x\left(\tau_{k}^{-}\right)\right)\right](s) d s
\end{aligned}
$$


It remains, by lemma 2.4, only to prove the assertion for operator $\Theta_{1}$

Step1. To verify that operator $\Theta_{1}$ satisfies property (S1). For any function $f, h \in C(J ; E)$, we have that

$$
\begin{aligned}
\|\left(\Theta_{1} f\right)(t) & -\left(\Theta_{1} h\right)(t) \| \\
& =\left\|\frac{1}{\Gamma(q)} \int_{0}^{t}(t-s)^{q-1} T(t-s) B W^{-1}\left[\frac{1}{\Gamma(q)} \int_{0}^{b}(b-\tau)^{q-1} T(b-\tau)(h(\tau)-f(\tau)) d \tau\right](s) d s\right\| \\
& \leq \tilde{M} \tilde{M}_{1} \frac{1}{\Gamma(q)} \int_{0}^{t}(t-s)^{q-1}\left\|W^{-1}\left[\frac{1}{\Gamma(q)} \int_{0}^{b}(b-\tau)^{q-1} T(b-\tau)(h(\tau)-f(\tau)) d \tau\right](s)\right\| d s \\
& \leq \tilde{M} \tilde{M}_{1} \frac{b^{q}}{\Gamma(q+1)} \cdot \sup _{s \in J}\left\|W^{-1}\left[\frac{1}{\Gamma(q)} \int_{0}^{b}(b-\tau)^{q-1} T(b-\tau)(h(\tau)-f(\tau)) d \tau\right](s)\right\| \\
& \leq \tilde{M} \tilde{M}_{1} \tilde{M}_{2} \frac{b^{q}}{\Gamma(q+1)}\left\|\frac{1}{\Gamma(q)} \int_{0}^{b}(b-\tau)^{q-1} T(b-\tau)(h(\tau)-f(\tau)) d \tau\right\| \\
& \leq \tilde{M}^{2} \tilde{M}_{1} \tilde{M}_{2} \frac{b^{q}}{\Gamma(q+1)} \frac{1}{\Gamma(q)} \int_{0}^{b}(b-\tau)^{q-1} d \tau \cdot \sup _{t \in J}\|h(\tau)-f(\tau)\| \\
& =\tilde{M}^{2} \tilde{M}_{1} \tilde{M}_{2}\left(\frac{b^{q}}{\Gamma(q+1)}\right)^{2}\|f-h\|_{C}
\end{aligned}
$$

From which it follows that $\left\|\Theta_{1} f-\Theta_{1} h\right\|_{C} \leq \tilde{M}^{2} \tilde{M}_{1} \tilde{M}_{2}\left(\frac{b^{q}}{\Gamma(q+1)}\right)^{2}\|f-h\|_{C}$

Step 2. To verify that operator $\Theta_{1}$ satisfies property (S2). Operator $\Theta_{1}$ may be alternatively Cast in the form

$$
\begin{aligned}
\left(\Theta_{1} f\right)(t):= & G\left(B W ^ { - 1 } \left[x_{1}-T(b)(\phi(0)-g(0, \phi))-g\left(b, y_{b}+\hat{\phi}_{b}\right)\right.\right. \\
& \left.\left.-\frac{1}{\Gamma(q)} \int_{0}^{b}(b-s)^{q-1} T(b-s) A g\left(s, y_{s}+\hat{\phi}_{s}\right) d s-\sum_{k=1}^{m} T\left(b-\tau_{k}\right) I_{k}\left(x\left(\tau_{k}^{-}\right)\right)-\theta G f\right]\right)
\end{aligned}
$$

Where $\theta: C(J ; E) \rightarrow E, \theta y=y(T)$ is a bounded linear operator? Then in view of lemma 2.4, the proof is completed.

From lemmas 2.3 and 3.1, we can conclude straightforwardly that:

Lemma 2.2 The multioperator $\Omega$ is u.s.c. and has compact-convex values.

Lemma 2.3 Under the condition

$$
\left(N+N^{2} N_{1} \frac{b^{q}}{\Gamma(q+1)} \sup _{s \in J} X(s)\right) \frac{b^{q}}{\Gamma(q+1)} \cdot \sup _{s \in J} k(s)<1
$$

The multioperator $\Omega$ is $\beta$-condensing, where positive constants $N, N_{1}$ are such that

$$
\sup _{t \in J}\|T(t)\|^{(X)} \leq N,\|B\|^{(X)} \leq N_{1} .
$$

Proof. Assume that $M \subset B_{v}$ is a bounded set satisfying 


$$
\beta(\Omega(M)) \geq \beta(M)
$$

In the sense of order generated by the cone $R_{+}^{2}$. It is sufficient to prove that $\mathrm{M}$ is a precompact set.

By virtue of lemma 2.5 and (H5), it is easy to show that

$$
X_{E}\left(G \circ P_{F}(M)(t)\right) \leq \frac{N \varphi(M)}{\Gamma(q)} \int_{0}^{t}(t-s)^{q-1} k(s) d s \leq \frac{N \varphi(M) b^{q}}{\Gamma(q+1)} \cdot \sup _{s \in J} k(s)
$$

Direct derivation, in view of the hypothesis (H6) and inequality (7), has that

$$
\begin{aligned}
X_{E} & \left(\left\{T ( t - s ) B W ^ { - 1 } \left[x_{1}-T(b)(\phi(0)-g(0, \phi))-g\left(b, y_{b}+\hat{\phi}_{b}\right)-\frac{1}{\Gamma(q)} \int_{0}^{b}(b-s)^{q-1} T(b-s) .\right.\right.\right. \\
& \left.\left.\left.\left(A g\left(s, y_{s}+\hat{\phi}_{s}\right)+f(s)\right) d s-\sum_{k=1}^{m} T\left(b-\tau_{k}\right) I_{k}\left(x\left(\tau_{k}^{-}\right)\right)\right](s): f \in P_{F}(M)\right\}\right) \\
\leq & N N_{1} X(s) X_{E}\left(\left\{\frac{1}{\Gamma(q)} \int_{0}^{b}(b-s)^{q-1} T(b-s) f(s) d s: \quad f \in P_{F}(M)\right\}\right) \\
\leq & \frac{N^{2} N_{1} X(s) b^{q}}{\Gamma(q+1)} \cdot \sup _{s \in J} k(s) \cdot \varphi(M)
\end{aligned}
$$

From which it follows that

$$
\begin{aligned}
X_{E}\left(\Theta_{1} \circ P_{F}(M)(t)\right) & \leq \frac{N^{2} N_{1} b^{q}}{\Gamma(q+1)} \cdot \sup _{s \in J} k(s) \cdot \varphi(M) \cdot \frac{1}{\Gamma(q)} \int_{0}^{t}(t-s)^{q-1} X(s) d s \\
& \leq N^{2} N_{1}\left(\frac{b^{q}}{\Gamma(q+1)}\right)^{2} \cdot \sup _{s \in J} k(s) \cdot \sup _{s \in J} X(s) \cdot \varphi(M)
\end{aligned}
$$

Since it is easy to obtain that

$$
\begin{aligned}
\Omega(M)(t) \subset & -T(t) g(0, \phi)+g\left(t, y_{t}+\hat{\phi}_{t}\right)+\frac{1}{\Gamma(q)} \int_{0}^{t}(t-s)^{q-1} T(t-s) A g\left(s, y_{s}+\hat{\phi}_{s}\right) d s \\
& +\sum_{0<\tau_{k}<t} T\left(t-\tau_{k}\right) I_{k}\left(y\left(\tau_{k}^{-}\right)+\hat{\phi}\left(\tau_{k}^{-}\right)\right)+G \circ P_{F}(M)(t)+\Theta_{1} \circ P_{F}(M)(t), \text { for any } t \in J
\end{aligned}
$$

We have, along with the equalities (7) and (9), that

$$
\begin{aligned}
X_{E}(\Omega(M)(t)) & \leq X_{E}\left(G \circ P_{F}(M)(t)\right)+X_{E}\left(\Theta_{1} \circ P_{F}(M)(t)\right) \\
& \leq\left(N+N^{2} N_{1} \frac{b^{q}}{\Gamma(q+1)} \sup _{s \in J} X(s)\right) \frac{b^{q}}{\Gamma(q+1)} \cdot \sup k(s) \cdot \varphi(M)
\end{aligned}
$$

Which, in turn, yields that $\varphi(\Omega(M)) \leq \gamma \varphi(M)$, where

$$
\gamma:=\left(N+N^{2} N_{1} \frac{b^{q}}{\Gamma(q+1)} \sup _{s \in J} X(s)\right) \frac{b^{q}}{\Gamma(q+1)} \cdot \sup _{s \in J} k(s)<1 \text {. The assertion } \varphi(M)=0 \text { follows from }
$$

the condition (5) along with the combination of (6) and (10). The remaining proof follows from the analogous techniques used in [6-10]. Before proceeding, we shall need the following assertion.

Lemma 2.4 Assume that $z^{\prime} \in \lambda \Omega(y)$ for some $0<\lambda \leq 1$, i.e., $z^{\prime}=\lambda \Theta(f)$, where $f \in P_{F}(y)$. Then The following relation holds: 


$$
\left\|z^{\prime}(t)\right\| \leq C_{1}\|\phi\|_{B_{v}}+C_{2}\left(\|y\|_{C}+\tilde{M}\|\phi(0)\|\right)+C_{3}\|f\|_{C}
$$

Where $C_{1}:=(\tilde{M}+1) \gamma_{g}+\frac{b^{q} \tilde{M}}{\Gamma(q+1)} \gamma_{g}^{\prime}, C_{2}:=l\left(\gamma_{g}+\frac{b^{q} \tilde{M}}{\Gamma(q+1)} \gamma_{g}^{\prime}\right)+\tilde{M} \gamma_{1}\left(\gamma_{1}:=\sum_{k=1}^{m} \gamma_{k}\right)$

$$
C_{3}:=\left(\tilde{M}+\tilde{M}^{2} \tilde{M}_{1} \tilde{M}_{2} \frac{b^{q}}{\Gamma(q+1)}\right) \frac{b^{q}}{\Gamma(q+1)}
$$

Proof. In view of lemma 2.1, we have that

$$
\begin{aligned}
z^{\prime}(t)= & -\lambda T(t) g(0, \phi)+\lambda g\left(t, y_{t}+\hat{\phi}_{t}\right)+\frac{\lambda}{\Gamma(q)} \int_{0}^{t}(t-s)^{q-1} T(t-s) A g\left(s, y_{s}+\hat{\phi}_{s}\right) d s \\
& +\lambda \sum_{0<\tau_{k}<t} T\left(t-\tau_{k}\right) I_{k}\left(y\left(\tau_{k}^{-}\right)+\hat{\phi}\left(\tau_{k}^{-}\right)\right)+\lambda(G f)(t)+\lambda\left(\Theta_{1} f\right)(t) \\
\leq & \tilde{M} \gamma_{g}\|\phi\|_{B_{v}}+\gamma_{g}\left\|y_{t}+\hat{\phi}_{t}\right\|_{B_{v}}+\frac{b^{q} \tilde{M}}{\Gamma(q+1)} \gamma_{g}^{\prime} \cdot \sup _{s \in J}\left\|y_{s}+\hat{\phi}_{s}\right\|_{B_{v}}+\tilde{M} \sum_{0<\tau_{k}<t} \gamma_{k}\left\|y\left(\tau_{k}^{-}\right)+\hat{\phi}\left(\tau_{k}^{-}\right)\right\| \\
& +\frac{b^{q} \tilde{M}}{\Gamma(q+1)}\|f\|_{C}+\tilde{M}^{2} \tilde{M}_{1} \tilde{M}_{2}\left(\frac{b^{q}}{\Gamma(q+1)}\right)^{2}\|f\|_{C} \\
\leq & C_{1}\|\phi\|_{B_{v}}+C_{2}\left(\|y\|_{C}+\tilde{M}\|\phi(0)\|\right)+C_{3}\|f\|_{C}
\end{aligned}
$$

Theorem 2.1 Under the conditions (H1)-(H6), suppose that there exists a constant $\mathrm{L}>0$ such that

$$
\frac{L}{C_{1}\|\phi\|_{B_{v}}+C_{2}(L+\tilde{M}\|\phi(0)\|)+C_{3}\|h\|_{C} \psi\left(\|\phi\|_{B_{v}}+l(L+\tilde{M}\|\phi(0)\|)\right)}>1
$$

Where the constants $C_{1}, C_{2}$ and $C_{3}$ are those given as in lemma 3.4. Then system Eq.(1)-Eq.(3) is controllable on the interval J.

Proof. Suppose that $y \in \lambda \Omega(y)$ for some $y \in B_{v}^{\prime \prime}$ and $0<\lambda \leq 1$, then $y=\lambda \Theta(f), f \in P_{F}(y)$. Using Lemma 3.4 and no decreasing property of function $\psi(\cdot)$, we can easily yield that

$$
\begin{aligned}
\|y\|_{C} & \leq C_{1}\|\phi\|_{B_{v}}+C_{2}\left(\|y\|_{C}+\tilde{M}\|\phi(0)\|\right)+C_{3}\|f\|_{C} \\
& \leq C_{1}\|\phi\|_{B_{v}}+C_{2}\left(\|y\|_{C}+\tilde{M}\|\phi(0)\|\right)+C_{3}\|h\|_{C} \psi\left(\|\phi\|_{B_{v}}+l(L+\tilde{M}\|\phi(0)\|)\right)
\end{aligned}
$$

If we take $V:=\left\{y \in B_{v}^{\prime \prime},\|y\|_{C}<L\right\}$, then from inequality (11), we have that $y \notin \lambda \Omega(y)$, for any $y \in \partial V$ And $0<\lambda \leq 1$, and moreover using lemmas 2.2,3.2 and 3.3, the proof is completed.

\section{Acknowledgements}

This work is partially supported by the National Nature Science Foundation of P.R.China under Grant contract 60574018. 


\section{References}

[1] R.Ye, Existence of solutions for impulsive partial neutral functional differential equation with infinite delay, Nonlinear Analysis, 73(2010)155-162

[2] J.Banas, On solutions of a neutral differential equation with deviating argument, Mathematical and Computer Modelling, 44(2006)1080-1088

[3] T.Cardinali, et al., On the existence of mild solutions of semilinear evolution differential inclusions, J.Math.Anal.Appl.,308(2005)620-635

[4] Y.K.Chang, Controllability of impulsive functional differential systems with infinite delay in Banach spaces, Chaos, Solitons and Fractals, 33(2007)1601-1609

[5] M. Kamenskii, et al., Condensing Multivalued Maps and Semilinear Differential Inclusions in Banach Spaces, in: de Gruyter Series in Nonlinear Analysis and Applications, vol.7, Walter de Gruyter, Berlin, New York, 2001

[6] V.Obukhovski, et al., Controllability for systems governed by semilinear differential inclusions in a Banach space with a noncompact semigroup, Nonlinear Analysis, 70(2009)3424-343

[7] J.Hale, S.M.V. Lunel, Introduction to functional differential equations, New York: Springer-Verlag, 1993

[8] K.S.Miller, B.Ross, An introduction to the fractional calculus and fractional differential equations, New York: Wiley, 1993

[9] D.R.Smart, Fixed point theorems, Cambridge: Cambridge University Press, 1980

[10] V.B.Kolmanovskii, Applied theory of functional differential equations, Boston: Kluwer Academic Publishers, 1992 